Das Parlament hat den Gesetzestext zum Zulassungsstopp revidiert - eine Bestimmung, auf deren negative Auswirkungen wir bereits mehrfach hingewiesen haben: Wir gehen deshalb nicht erneut darauf ein. Zur Ausführungsverordnung wurde nun eine Vernehmlassung eröffnet, für die wir unsererseits alle Gesellschaften der
FMH angehört haben. Hanspeter Kuhn und Gabriela Lang vom Rechtsdienst legen nachstehend unsere Antwort an den Bundesrat dar.

Dr. med. Jacques de Haller, Präsident der FMH

\title{
Zulassungsstopp: Revision der Verordnung
}

Im Juni 2009 hat das Schweizer Parlament entschieden, den Zulassungsstopp nochmals um zwei Jahre zu verlängern mit Änderungen: Weiterhin unter den Zulassungsstopp fallen alle Spezialistinnen und Spezialisten; ausgenommen sind ab 2010 Ärztinnen und Ärzte, die in ihrer Weiterbildung ausschliesslich einen Grundversorgertitel erworben haben [1]. Der Bundesrat hat den Entwurf für die aktualisierte Ausführungsverordnung (neues Kürzel: VEZL) in Vernehmlassung gegeben. In ihrer Antwort weist die FMH auf zwei zentrale Punkte hin, die bei der Aktualisierung des Verordnungstextes zu berücksichtigen sind:

- Arztpraxen und Gruppenpraxen sind rechtsgleich zu behandeln.

- Anstatt Höchstzahlen sind Richtzahlen vorzuziehen.

Die Ausführungsverordnung des Bundes soll den revidierten Gesetzestext von Art. 55a umsetzen. Das Gesetz regelt die selbstständig und unselbständig in Arztpraxen tätigen Ärzte (diejenigen in Gruppenpraxen wie z. B. HMO gemäss Art. 36a KVG) und die ambulant am Spital tätigen, gleich: Ihre Arbeit zulasten der obligatorischen Krankenpflegeversicherung kann von einem Bedürfnis abhängig gemacht werden. Der Gesetzgeber wollte insbesondere die Ärzte in normalen Arztpraxen und solche in Einrichtungen gemäss Art. 36a KVG gleich behandeln. Er hat im Jahr 2008 Art. 55a KVG explizit ergänzt, um die vorher bestehende Ungleichbehandlung (Zulassung der sos-médecins in Genf) zu korrigieren [2]. Damit sind Bundesrat und Kantone verpflichtet, Ärzte in normalen Praxen und solche in Gruppenpraxen gleich zu behandeln die Zulassung in einem Kanton hängt für beide vom Bedürfnis $a b$, oder sie ist für beide frei.

\section{Arztpraxen und Gruppenpraxen rechtsgleich behandeln}

Weil der Gesetzgeber gewöhnliche Arztpraxen und Gruppenpraxen gleich behandeln will, soll auch der Bundesrat in der Ausführungsverordnung die Regelung einheitlich formulieren, getreu der Juristenregel, dass gleich geschrieben wird, was gleich gemeint ist. Die FMH erachtet die für die Institutionen nach Art. 36a KVG und die Spitalambulanz vorgeschlagene Formulierung als klar und ehrlich: «Die Kantone können die Tätigkeit (...) von einem Bedürfnis abhängig machen.»(Art. 1a Abs. 1 Entwurf VEZL).
Wenn die Kantone richtigerweise für alle drei Kategorien von Leistungserbringern erheblichen Ermessensspielraum haben sollen, spricht eine klare und transparente Regelung in der Verordnung konsequenterweise von «Richtzahlen» und nicht von «Höchstzahlen». Der Bund soll solche Zahlen als unverbindliche Planungshilfe an die Kantone veröffentlichen.

Wollte der Gesetzgeber tatsächlich, dass der Bundesrat Höchstzahlen festlegt - der Gesetzestext spricht bekanntlich von «Kriterien» und nicht von Höchstzahlen - müssten diese unter dem Aspekt der Verteilungsgerechtigkeit vertretbar sein. Weshalb sollten denn z. B. die Walliserinnen nur 9,9 Gynäkologinnen und Gynäkologen pro hunderttausend Einwohner aufsuchen können, die Genferinnen hingegen 29,4? Und wie sollte unter dem Aspekt der Verteilungsgerechtigkeit vertreten werden, dass die Appenzeller und St. Galler Patienten

\section{Richtzahlen statt Höchstzahlen}

Zugang zu 19 (AR), 0 (AI) bzw. 16,2 (SG) Psychiaterinnen und Psychiatern pro 100000 Einwohner haben, die Basler hingegen zu 82,3 (BS)?

Schliesslich regt die FMH an, dass die Kantone auch die Anzahl der Grundversorgerärztinnen und -ärzte erfassen und dem BAG melden, auch wenn sie dem Zulassungsstopp nicht mehr unterstellt sind. Die Kantone können auf diese Informationen zurückgreifen, wenn es beispielsweise gilt, eine Unterversorgung im Kanton zu erkennen und mit Anreizen zu beheben.

Hanspeter Kuhn, stv. Generalsekretär FMH Gabriela Lang, Rechtsdienst FMH

\section{Literatur}

1 Wortlaut Art. 55a KVG von 1.1.2010 bis 31.12.2011 (Auszug): «Davon (vom Zulassungsstopp; Anm. d. Autoren) ausgenommen sind Personen mit folgendem Weiterbildungstitel: a. Allgemeinmedizin; b. Praktischer Arzt oder praktische Ärztin als einziger Weiterbildungstitel; c. Innere Medizin als einziger Weiterbildungstitel; d. Kinder- und Jugendmedizin.»

2 Vgl. Kuhn HP. Zulassungsstopp verlängert und auf Gruppenpraxen ausgeweitet. Schweiz Ärztezeitung. 2008; 89(26/27):1169. 